\title{
ON THE ASYMPTOTIC BEHAVIOUR OF AN ALGEBROID FUNCTION OF ORDER ZERO
}

\author{
Tsuneo SATO
}

(Received 11 September 2000, revised 15 February 2001 and accepted 8 March 2001)

\section{Introduction}

In this paper we shall investigate a relation between the maximum modulus and the minimum modulus of an algebroid function of order zero. We denote the Nevanlinna deficiency and the Valiron deficiency of value $a$ for $f(z)$ by $\delta(a, f)$ and $\Delta(a, f)$, respectively (see $[4,5,8,9,17]$ ).

We denote the maximum modulus $M(r, f)$ and the minimum modulus $L(r, f)$ of a meromorphic function $f(z)$ by

$$
\begin{aligned}
M(r, f) & =\sup _{|z|=r}|f(z)|, \\
L(r, f) & =\inf _{|z|=r}|f(z)|,
\end{aligned}
$$

respectively. We shall assume that $f(z)$ is transcendental, i.e. that

$$
\log r=o(T(r, f)) \quad(r \rightarrow \infty) .
$$

If $E$ is a measurable set on $(0, \infty)$ we define its density as

$$
\lim _{r \rightarrow \infty} \frac{m(E \cap(0, r))}{r}
$$

if the limit exists, and its upper density by replacing $\lim$ by $\lim \sup$, where $m$ ( $E \cap$ $(0, r))$ denotes the measure of $E \cap(0, r)$.

It is well known that if $f(z)$ is an entire function of order zero, then

$$
\log L(r, f) \sim \log M(r, f) \sim T(r, f)
$$

in a set of $r$ of upper density 1 [3]. Kubota [7] showed the following striking and elegant theorem which is an analogous result for a meromorphic function of order zero. 
THEOREM. Let $f(z)$ be a meromorphic function of order zero. If $\delta(\infty, f)>0$ then

$$
\log L(r, f) \sim \log M(r, f)
$$

and

$$
\delta(\infty, f) \leq \liminf _{r \rightarrow \infty} \frac{\log L(r, f)}{T(r, f)} \leq \limsup _{r \rightarrow \infty} \frac{\log M(r, f)}{T(r, f)} \leq \Delta(\infty, f)
$$

in a set of $r$ of upper density 1 . Hence if $\delta(\infty, f)=\Delta(\infty, f)>0$, then

$$
\log L(r, f) \sim \log M(r, f) \sim \Delta(\infty, f) T(r, f)
$$

in a set of $r$ of upper density 1.

The purpose of this paper is to extend Kubota's theorem to $n$-valued algebroid functions of order zero. Let $f(z)$ be an $n$-valued algebroid function defined by an irreducible equation

$$
F(z, f) \equiv A_{0}(z) f^{n}+A_{1}(z) f^{n-1}+\cdots+A_{n-1}(z) f+A_{n}(z)=0,
$$

where $A_{0}, A_{1}, \ldots, A_{n}$ are entire functions without common zeros. Let $a_{1}, \ldots, a_{n}$ be complex numbers, different from each other.

We put

$$
\begin{aligned}
g_{v}(z) & =F\left(z, a_{v}\right) \quad(v=1,2, \ldots, n) \\
g(z) & =\max _{1 \leq v \leq n}\left\{1,\left|g_{v}(z)\right|\right\}
\end{aligned}
$$

where $F(z, f)=0$ is the defining equation of $f$.

We put

$$
f_{v}(z)=\frac{g_{v}(z)}{A_{0}(z)}
$$

and set

$$
\begin{aligned}
M(r, f) & =\sup _{|z|=r} \max _{1 \leq v \leq n}\left|f_{v}(z)\right|, \\
L(r, f) & =\inf _{|z|=r} \max _{1 \leq v \leq n}\left|f_{v}(z)\right| .
\end{aligned}
$$

We shall prove the following extension of Kubota's theorem which gives a precise meaning to the asymptotic behaviour of an $n$-valued algebroid function of order zero.

THEOREM 1. Let $f(z)$ be an $n$-valued transcendental algebroid function of order zero defined by (1). 
If $\delta(\infty, f)>0$, then

$$
\log L(r, f) \sim \log M(r, f)
$$

and

$$
\delta(\infty, f) \leq \liminf _{r \rightarrow \infty} \frac{\log L(r, f)}{T(r, f)} \leq \limsup _{r \rightarrow \infty} \frac{\log M(r, f)}{T(r, f)} \leq n \Delta(\infty, f)
$$

in a set of $r$ of upper density 1.

Hence, if $\delta(\infty, f)=\Delta(\infty, f)>0$ and $n=1$, then

$$
\log L(r, f) \sim \log M(r, f) \sim \Delta(\infty, f) T(r, f)
$$

in a set of $r$ of upper density 1.

THEOREM 2. Let $f(z)$ be an n-valued transcendental algebroid function of order zero defined by (1).

$$
\text { If } \delta(\infty, f)>0 \text {, then }
$$

$$
\log L(r, f) \sim \log M(r, f)
$$

and

$$
\max _{v} \delta\left(\infty, f_{v}\right) \leq \liminf _{r \rightarrow \infty} \frac{\log L(r, f)}{T(r, f)} \leq \liminf _{r \rightarrow \infty} \frac{\log M(r, f)}{T(r, f)} \leq n^{2} \max _{v} \delta\left(\infty, f_{v}\right)
$$

in a set of $r$ of upper density 1.

THEOREM 3. Under the same conditions of Theorem 1, we have

$$
\max _{v} \Delta\left(\infty, f_{v}\right) \leq \limsup _{r \rightarrow \infty} \frac{\log L(r, f)}{T(r, f)} \leq \limsup _{r \rightarrow \infty} \frac{\log M(r, f)}{T(r, f)} \leq n \max _{v} \Delta\left(\infty, f_{v}\right)
$$

in a set of $r$ of upper density 1.

COROLLARY 1. Under the same conditions of Theorem 1, we have

$$
\delta(\infty, f) \leq \liminf _{r \rightarrow \infty} \frac{\log L(r, f)}{T(r, f)} \leq \liminf _{r \rightarrow \infty} \frac{\log M(r, f)}{T(r, f)} \leq n \delta(\infty, f)
$$

in a set of $r$ of upper density 1.

COROLLARY 2. Under the same conditions of Theorem 1, we have

$$
\frac{1}{n} \Delta(\infty, f) \leq \limsup _{r \rightarrow \infty} \frac{\log L(r, f)}{T(r, f)} \leq \limsup _{r \rightarrow \infty} \frac{\log M(r, f)}{T(r, f)} \leq n \Delta(\infty, f)
$$

in a set of $r$ of upper density 1. 


\section{Preliminaries}

Let $f(z)$ be an $n$-valued transcendental algebroid function defined by an irreducible equation (1). Let $y_{j}(z)$ be the $j$ th determination of $f(z), 1 \leq j \leq n$, and

$$
\mu(r, A)=\frac{1}{2 \pi n} \int_{0}^{2 \pi} \log A\left(r e^{i \theta}\right) d \theta,
$$

where

$$
A(z)=\max _{0 \leq \nu \leq n}\left\{1,\left|A_{v}(z)\right|\right\} .
$$

Then, by a theorem of Valiron [16], we have

$$
\mu(r, A)=T(r, f)+O(1) .
$$

Ozawa [10] showed that

$$
\mu(r, g)=\mu(r, A)+O(1)
$$

and

$$
\sum_{j=1}^{n} \log ^{+}\left|y_{j}(z)\right| \leq \log \left|\frac{A(z)}{A_{0}(z)}\right|+O(1) .
$$

On the other hand, Tsumura [15] showed that

$$
\sum_{j=1}^{n} \log ^{+}\left|y_{j}(z)\right| \geq \log \left|\frac{A(z)}{A_{0}(z)}\right|-O(1) .
$$

In order to prove our theorem, we need the following lemmas. Lemmas 1 and 2 are essentially the same as lemmas in Boas [2] and Cartwright [3], hence we omit their proofs.

LEMMA 1. If $\varphi(z)$ is a meromorphic function of order less than one with $\varphi(0)=1$, then for every $\eta\left(0<\eta<\frac{8}{3} e\right)$ we have

$$
\begin{aligned}
& |\log | \varphi(z)|-\{N(2 R, 0 ; \varphi)-N(2 R, \infty ; \varphi)\}| \\
& \quad<\left(1+\log \frac{4 e}{\eta}\right)\{Q(2 R, 0 ; \varphi)+Q(2 R, \infty ; \varphi)\}
\end{aligned}
$$

in $|z|<R$, outside a set of circles the sum of whose radii is at most $2 \eta R$, where

$$
Q(r, a ; \varphi)=r \int_{r}^{\infty} \frac{n(t, a ; \varphi)}{t^{2}} d t .
$$


LEMMA 2. If $\varphi(z)$ is of order zero, then

$$
\liminf _{r \rightarrow \infty} \frac{Q(r, 0 ; \varphi)+Q(r, \infty ; \varphi)}{N(r)}=0,
$$

where $N(r)=N(r, 0 ; \varphi)+N(r, \infty ; \varphi)$.

The following lemma plays an important role for the proof of our theorem.

LEMMA 3. Let $f(z)$ be an n-valued transcendental algebroid function defined by (1). Then we have

$$
m(r, f) \leq \frac{1}{n} \sum_{\nu=1}^{n} m\left(r, f_{v}\right)+O(1) .
$$

On the other hand we have

$$
m(r, f) \geq \max _{v} \frac{1}{n} m\left(r, f_{v}\right)-O(1) .
$$

Proof. First we can consider

$$
g_{v}(z)=\sum_{j=0}^{n} a_{v}^{j} A_{n-j}(z), \quad v=1,2, \ldots, n
$$

as a system of linear equations in $A_{1}(z), \ldots, A_{n}(z)$.

Hence we can solve from (20)

$$
A_{v}(z)=\sum_{i=1}^{n} \alpha_{v, i} g_{i}(z)+\alpha_{v, 0} A_{0}(z), \quad v=1,2, \ldots, n
$$

where $\alpha_{v, i}$ are constants.

Then we obtain

$$
\begin{aligned}
\log \left|\frac{A}{A_{0}}\right| & =\log \left(\max _{1 \leq \nu \leq n}\left|\frac{A_{v}}{A_{0}}\right|\right) \\
& \leq \log \left(\sum_{\nu=1}^{n}\left|\frac{g_{v}}{A_{0}}\right|\right)+O\left(\log \sum_{\nu=0}^{n}\left|\alpha_{\nu, i}\right|\right) \\
& =\log \left(\sum_{\nu=1}^{n}\left|f_{\nu}\right|\right)+O(1) \\
& \leq \sum_{\nu=1}^{n} \log ^{+}\left|f_{\nu}\right|+O(1) .
\end{aligned}
$$


Thus we have, in view of (14),

$$
\begin{aligned}
m(r, f) & =\frac{1}{2 n \pi} \int_{0}^{2 \pi} \sum_{j=1}^{n} \log ^{+}\left|y_{j}\left(r e^{i \theta}\right)\right| d \theta \\
& \leq \frac{1}{2 n \pi} \int_{0}^{2 \pi} \sum_{\nu=1}^{n} \log ^{+}\left|f_{v}\left(r e^{i \theta}\right)\right| d \theta+O(1) \\
& =\frac{1}{n} \sum_{\nu=1}^{n} m\left(r, f_{\nu}\right)+O(1)
\end{aligned}
$$

which gives (18).

Next we use the following inequality:

$$
\max _{v} \log \left|g_{v}\right| \leq \max _{v} \log \left|\sum_{j=0}^{n} A_{n-j} a_{v}^{j}\right| \leq \log A+O(1),
$$

which follows from (20); then we have, by using (15),

$$
\begin{aligned}
m(r, f) & =\frac{1}{2 n \pi} \int_{0}^{2 \pi} \sum_{j=1}^{n} \log ^{+}\left|y_{j}\left(r e^{i \theta}\right)\right| d \theta \\
& \geq \frac{1}{2 n \pi} \int_{0}^{2 \pi} \log \left|\frac{A\left(r e^{i \theta}\right)}{A_{0}\left(r e^{i \theta}\right)}\right| d \theta-O(1) \\
& =\frac{1}{2 n \pi} \int_{0}^{2 \pi} \log ^{+}\left|\frac{A\left(r e^{i \theta}\right)}{A_{0}\left(r e^{i \theta}\right)}\right| d \theta-O(1) \\
& \geq \frac{1}{2 n \pi} \int_{0}^{2 \pi} \max _{\nu} \log ^{+}\left|\frac{g_{v}\left(r e^{i \theta}\right)}{A_{0}\left(r e^{i \theta}\right)}\right| d \theta-O(1) \\
& \geq \max _{1 \leq v \leq n} \frac{1}{n} m\left(r, f_{\nu}\right)-O(1)
\end{aligned}
$$

which gives (19). This completes the proof of Lemma 3.

By using Lemma 3 we have the following lemma which is analogous to the results of Selberg [14]:

LEMMA 4. Let $f(z)$ and $f_{v}(z)(v=1,2, \ldots, n)$ be defined in (1) and (2), respectively. Then we have

$$
\frac{1}{n} T\left(r, f_{v}\right)-O(1)<T(r, f)<\frac{1}{n} \sum_{\nu=1}^{n} T\left(r, f_{v}\right)+O(1) .
$$


Proof. In view of (19), we have

$$
\begin{aligned}
T(r, f) & =m(r, f)+N(r, f) \\
& \geq \frac{1}{n} m\left(r, f_{v}\right)-O(1)+N\left(r, 1 / A_{0}\right) \\
& >\frac{1}{n}\left\{m\left(r, f_{v}\right)+N\left(r, f_{v}\right)\right\}-O(1) \\
& =\frac{1}{n} T\left(r, f_{v}\right)-O(1) .
\end{aligned}
$$

Also because of (18), we obtain

$$
\begin{aligned}
T(r, f) & \leq \frac{1}{n} \sum_{\nu=1}^{n} m\left(r, f_{v}\right)+O(1)+N\left(r, 1 / A_{0}\right) \\
& =\frac{1}{n} \sum_{\nu=1}^{n} m\left(r, f_{v}\right)+\frac{1}{n} \sum_{\nu=1}^{n} N\left(r, f_{v}\right)+O(1) \\
& =\frac{1}{n} \sum_{\nu=1}^{n} T\left(r, f_{v}\right)+O(1),
\end{aligned}
$$

so the lemma is proved.

\section{The relation between $\delta(\infty, f)$ and $\delta\left(\infty, f_{v}\right)$ or $\Delta(\infty, f)$ and $\Delta\left(\infty, f_{v}\right)$}

We shall survey a relation between $\delta(\infty, f)$ and $\delta\left(\infty, f_{v}\right)$, and also a relation between $\Delta(\infty, f)$ and $\Delta\left(\infty, f_{v}\right)$.

LEMMA 5. Let $f(z)$ be an n-valued transcendental algebroid function defined by an irreducible equation (1). Then we have

$$
\begin{gathered}
\delta(\infty, f) \geq \delta\left(\infty, f_{v}\right) \quad(v=1,2, \ldots, n), \\
\Delta(\infty, f) \geq \Delta\left(\infty, f_{v}\right) \quad(v=1,2, \ldots, n) .
\end{gathered}
$$

Proof. By making some mention of the conception of Valiron [16], and using (12), we have

$$
\begin{aligned}
\delta(\infty, f) & =\liminf _{r \rightarrow \infty} \frac{m(r, f)}{T(r, f)}=\liminf _{r \rightarrow \infty}\left(1-\frac{N(r, f)}{T(r, f)}\right) \\
& =\liminf _{r \rightarrow \infty}\left(1-\frac{N\left(r, 1 / A_{0}\right)}{n \mu(r, A)}\right) .
\end{aligned}
$$


In view of (21), we obtain

$$
\delta(\infty, f) \geq \liminf _{r \rightarrow \infty}\left(1-\frac{N\left(r, 1 / A_{0}\right)}{T\left(r, f_{v}\right)}\right)=\delta\left(\infty, f_{v}\right) \quad(v=1,2, \ldots, n),
$$

which gives (22).

Similarly we can deduce (23), which gives the validity of Lemma 5.

The following lemma shows an essential relation of deficiencies between $\delta(\infty, f)$ and $\delta\left(\infty, f_{v}\right)$.

LEMMA 6. Let $f(z)$ be an n-valued algebroid function defined by (1). Then we have some suitable functions $\left\{f_{v}\right\}$ such as

$$
\delta(\infty, f) \leq n \delta\left(\infty, f_{v}\right) \quad(v=1,2, \ldots, n) .
$$

Proof. Let $\mathfrak{A}=\left(A_{0}(z), \ldots, A_{n}(z)\right)$ be a holomorphic curve. For $B=\left(\beta_{0}, \ldots, \beta_{n}\right)$ $\in \mathbb{P}^{n}$, we have [18, p 93 Theorem 4.5],

$$
N(r, B)+m(r, B)=T(r, \mathfrak{A})+O(1) .
$$

Hence we have $N(r, B) \sim T(r, \mathfrak{A})$ for $B \notin \mathcal{B}$, where $\mathcal{B}$ is the set of Valiron deficiencies which is $\mathcal{P}$-polar† in the projective space of hyperplanes in $\mathbb{P}^{n}$ in view of $[6,12]$.

Suppose $B_{j}=\left(a_{j}^{n}, a_{j}^{n-1}, \ldots, a_{j}, 1\right) \notin \mathcal{B}, 1 \leq j \leq n$. Then

$$
m\left(r, B_{j}\right)=o\left(N\left(r, B_{j}\right)\right)=o(T(r, \mathfrak{A})) .
$$

Write

$$
h_{j}(z)=a_{j}^{n-1} A_{1}(z)+\cdots+A_{n}(z) \equiv g_{j}(z)-a_{j}^{n} A_{0}(z) .
$$

Then, writing

$$
\mathbb{A}_{j}=\left\|B_{j}\right\|=\sqrt{\sum_{i=0}^{n}\left|a_{j}^{n-i}\right|^{2}}
$$

$\dagger$ A set $E \subset D$ is called $\mathcal{P}$-polar if it is the set of singular points of some plurisuperharmonic function on $D$ which is not identically $+\infty$. 


$$
\begin{aligned}
m\left(r, B_{j}\right)= & \frac{1}{2 \pi} \int_{0}^{2 \pi} \log \frac{\|\mathfrak{A}\|}{\left|g_{j}(z)\right|} d \theta \\
= & \frac{1}{2 \pi} \int_{0}^{2 \pi} \log \frac{\mathbb{A}_{j} \sqrt{1+\left|A_{1}(z) / A_{0}(z)\right|^{2}+\cdots+\left|A_{n}(z) / A_{0}(z)\right|^{2}}}{\left|f_{j}(z)\right|} d \theta \\
& +\log \frac{1}{\mathbb{A}_{j}} .
\end{aligned}
$$

Thus

$$
m\left(r, \frac{1}{f_{j}}\right)=\frac{1}{2 \pi} \int_{0}^{2 \pi} \log ^{+}\left|\frac{1}{f_{j}(z)}\right| d \theta \leq m\left(r, B_{j}\right)+O(1) .
$$

Suppose, for each $j, 1 \leq j \leq n, A_{0}(z)$ and $h_{j}(z)$ have no common zeros.

$$
\begin{aligned}
N\left(r, 1 / f_{j}\right)+m\left(r, 1 / f_{j}\right)+O(1) & =N\left(r, f_{j}\right)+m\left(r, f_{j}\right)=T\left(r, f_{j}\right), \\
N\left(r, 1 / f_{j}\right) & =N\left(r, B_{j}\right) \sim T(r, \mathfrak{A}), \\
m\left(r, 1 / f_{j}\right) & \leq m\left(r, B_{j}\right)=o\left(N\left(r, B_{j}\right)\right), \\
N\left(r, f_{j}\right) & =N\left(r, 1 / A_{0}\right) .
\end{aligned}
$$

Thus

$$
m\left(r, f_{j}\right) \sim T(r, \mathfrak{A})-N\left(r, 1 / A_{0}\right) \sim m\left(r, f_{j^{\prime}}\right)
$$

for any $j, j^{\prime}$.

On the other hand, we have

$$
\begin{aligned}
& m(r, f) \leq \frac{1}{n} \sum_{j=1}^{n} m\left(r, f_{j}\right)+O(1), \\
& T\left(r, f_{j}\right) \leq n T(r, f)+O(1) .
\end{aligned}
$$

Thus we have

$$
\begin{aligned}
\frac{m(r, f)}{T(r, f)} & \leq \sum_{j=1}^{n} \frac{m\left(r, f_{j}\right)}{n T(r, f)} \\
& \sim \frac{n m\left(r, f_{j}\right)}{n T(r, f)}(1+o(1)) \leq \frac{n m\left(r, f_{j}\right)}{T\left(r, f_{j}\right)} \quad(1 \leq j \leq n) .
\end{aligned}
$$

Hence we deduce

$$
\delta(\infty, f) \leq n \delta\left(\infty, f_{v}\right)
$$

for all $v$. 
LEMMA 7. Let $f(z)$ be an $n$-valued algebroid function defined by (1). Then we have

$$
\Delta(\infty, f) \leq n \max _{v} \Delta\left(\infty, f_{v}\right)
$$

Proof. In view of (21) and (18), we have

$$
\begin{aligned}
\Delta(\infty, f) & =\limsup _{r \rightarrow \infty} \frac{m(r, f)}{T(r, f)} \leq \limsup _{r \rightarrow \infty} \frac{\frac{1}{n} \sum_{\nu=1}^{n} m\left(r, f_{v}\right)+O(1)}{T(r, f)} \\
& =\limsup _{r \rightarrow \infty} \frac{1}{n} \sum_{\nu=1}^{n} \frac{m\left(r, f_{v}\right)}{T(r, f)} \leq \limsup _{r \rightarrow \infty} \frac{1}{n} \sum_{\nu=1}^{n} \frac{m\left(r, f_{v}\right)}{(1 / n) T\left(r, f_{v}\right)-O(1)} \\
& \leq \sum_{\nu=1}^{n} \limsup _{r \rightarrow \infty} \frac{m\left(r, f_{v}\right)}{T\left(r, f_{v}\right)}=\sum_{\nu=1}^{n} \Delta\left(\infty, f_{v}\right) \\
& \leq n \max _{v} \Delta\left(\infty, f_{v}\right) .
\end{aligned}
$$

The following lemma announces that our definitions of the maximum modulus $M(r, f)$ and the minimum modulus $L(r, f)$ of an algebroid function would seem to be independent of the selection of $\left\{a_{\nu}\right\}$ in view of (2)-(4).

LEMMA 8. Let $\left\{a_{\nu}\right\},\left\{b_{\nu}\right\}$ be distinct complex numbers of two sets. We put

$$
g_{v}(z)=F\left(z, a_{v}\right), \quad g_{v}^{*}(z)=F\left(z, b_{v}\right) \quad(v=1,2, \ldots, n)
$$

where $F(z, f)=0$ is the defining equation of $f$.

We put

$$
f_{v}(z)=\frac{g_{v}(z)}{A_{0}(z)}, \quad f_{v}^{*}(z)=\frac{g_{v}^{*}(z)}{A_{0}(z)}
$$

Then we have

$$
\begin{aligned}
\log M\left(r, f^{*}\right) & =\log M(r, f)+O(1), \\
\log L\left(r, f^{*}\right) & =\log L(r, f)+O(1) .
\end{aligned}
$$

Proof. By the definition we consider

$$
g_{v}(z)=\sum_{j=0}^{n} a_{v}^{j} A_{n-j}(z) \quad(v=1,2, \ldots, n),
$$

so we can solve

$$
A_{v}(z)=\sum_{i=1}^{n} \alpha_{v, i} g_{i}(z)+\alpha_{v, 0} A_{0}(z) \quad(v=1,2, \ldots, n)
$$


where $\alpha_{v, i}$ are constants. Similarly we have

$$
g_{v}^{*}(z)=\sum_{j=0}^{n} b_{v}^{j} A_{n-j}(z) \quad(v=1,2, \ldots, n),
$$

and

$$
A_{v}(z)=\sum_{i=1}^{n} \beta_{v, i} g_{i}^{*}(z)+\beta_{v, 0} A_{0}(z) \quad(v=1,2, \ldots, n)
$$

where $\beta_{v, i}$ are constants.

Thus we have from (3)

$$
\begin{aligned}
\log M(r, f) & =\sup _{|z|=r} \max _{v} \log \left|f_{v}\right| \\
& =\sup _{|z|=r} \max _{v} \log \left|\frac{g_{v}}{A_{0}}\right| \\
& \leq \sup _{|z|=r} \max _{v} \log \left(\sum_{v=0}^{n}\left|\frac{A_{v}}{A_{0}}\right|\right)+O\left(\log \sum_{v=1}^{n}\left|a_{v}^{j}\right|\right) \\
& \leq \sup _{|z|=r} \log \left(\max _{v}\left|\frac{A_{v}}{A_{0}}\right|\right)+O(1) \\
& =\sup _{|z|=r} \log \left(\max _{v}\left|\frac{\sum_{i=1}^{n} \beta_{v, i} g_{i}^{*}(z)+\beta_{v, 0} A_{0}(z)}{A_{0}}\right|\right)+O(1) \\
& \leq \sup _{|z|=r} \log \left(\max _{v}\left|\frac{g_{v}^{*}}{A_{0}}\right|\right)+O(1) \\
& =\sup _{|z|=r} \log \left(\max _{v}\left|f_{v}^{*}\right|\right)+O(1) \\
& =\sup _{|z|=r} \max _{v} \log \left|f_{v}^{*}\right|+O(1) \\
& =\log M\left(r, f^{*}\right)+O(1)
\end{aligned}
$$

The same reasoning yields

$$
\log M\left(r, f^{*}\right) \leq \log M(r, f)+O(1) .
$$

Thus we obtain

$$
\log M\left(r, f^{*}\right)=\log M(r, f)+O(1) .
$$

Similarly we have

$$
\log L\left(r, f^{*}\right)=\log L(r, f)+O(1) .
$$


Further, we shall show a relation between the maximum modulus of $M(r, f)$ of ours and the $M(r, y)$ introduced by Rauch [11] (see [13]) in the following.

LEMMA 9. Let

$$
\begin{aligned}
& \log M(r, y)=\sup _{|z|=r} \max _{v} \log \left|y_{v}(z)\right|, \\
& \log L(r, y)=\inf _{|z|=r} \max _{v} \log \left|y_{v}(z)\right|,
\end{aligned}
$$

which are both definitions of Valiron [16], are independent of $\left\{a_{\nu}\right\}$ entirely.

Then we have

$$
\begin{aligned}
\log M(r, y)-O(1) & \leq \log M(r, f) \leq n \log M(r, y)+O(1) \\
\log L(r, y)-O(1) & \leq \log L(r, f) \leq n \log L(r, y)+O(1)
\end{aligned}
$$

Proof. First we have, in view of (14), (15) and (3),

$$
\begin{aligned}
\log M(r, y) & =\sup _{|z|=r} \max _{\nu} \log \left|y_{v}(z)\right| \\
& \leq \sup _{|z|=r} \sum_{\nu=1}^{n} \log \left|y_{v}(z)\right| \\
& \leq \sup _{|z|=r} \sum_{\nu=1}^{n} \log ^{+}\left|y_{v}(z)\right| \leq \sup _{|z|=r} \log \left|\frac{A(z)}{A_{0}(z)}\right|+O(1) \\
& =\sup _{|z|=r} \log \left(\max _{v}\left|\frac{A_{v}}{A_{0}}\right|\right)+O(1) \\
& \leq \sup _{|z|=r} \log \left(\sum_{\nu=1}^{n}\left|\frac{g_{v}}{A_{0}}\right|\right)+O\left(\log \sum_{\nu=0}^{n}\left|\alpha_{v, i}\right|\right) \\
& =\sup _{|z|=r} \log \left(\sum_{\nu=1}^{n}\left|f_{v}\right|\right)+O(1) \\
& \leq \sup _{|z|=r} \log \left(n \max _{v}\left|f_{v}\right|\right)+O(1) \\
& \leq \sup _{|z|=r} \log \left(\max _{v}\left|f_{v}\right|\right)+O(1) \\
& =\sup _{|z|=r} \max _{v} \log \left|f_{v}\right|+O(1)=\log M(r, f)+O(1) .
\end{aligned}
$$


Next we have, by using (20),

$$
\begin{aligned}
\log M(r, f) & =\sup _{|z|=r} \max _{\nu} \log \left|f_{v}\right|=\sup _{|z|=r} \max _{\nu} \log \left|\frac{g_{v}}{A_{0}}\right| \\
& \leq \sup _{|z|=r} \log \left|\sum_{0}^{n} \frac{A_{n-j} a_{v}^{j}}{A_{0}}\right| \leq \sup _{|z|=r} \log \left|\frac{A}{A_{0}}\right|+O(1) \\
& \leq \sup _{|z|=r} \sum_{v=1}^{n} \log ^{+}\left|y_{v}\right|+O(1) \\
& \leq \sup _{|z|=r}\left(n \max _{v} \log \left|y_{v}\right|\right)+O(1) \\
& =n \log M(r, y)+O(1) .
\end{aligned}
$$

Thus we deduce the inequality (27).

Similarly we can deduce (28), which gives the validity of Lemma 8.

\section{Proof of Theorem 1}

Applying Lemma 1 for $\varphi(z)=f_{v}(z)$ and putting the right-hand side of (17) as $Q_{0}^{(v)}(2 R)$, we have

$$
Q_{0}^{(\nu)}(2 R)=Q\left(2 R, 0, f_{v}\right)+Q\left(2 R, \infty, f_{v}\right) .
$$

First we assume that each meromorphic function $f_{v}(z)(v=1,2, \ldots, n)$ satisfies

$$
T\left(r, f_{v}\right) \sim N_{v}\left(r, 0, f_{v}\right), \quad f_{v}(0)=1 .
$$

Suppose $\delta(\infty, f)>0$, then we can say that there exists some suitable functions $f_{v}(1 \leq v \leq n)$, such that $\delta\left(\infty, f_{v}\right)>0$ in view of (24), so that there is for some positive numbers $\rho_{v}(v=1, \ldots, n), 0<\rho_{v}<\delta\left(\infty, f_{v}\right)$, and $R_{0}^{(v)}$, by the definition of deficiency

$$
N\left(2 R, 0, f_{v}\right)-N\left(2 R, \infty, f_{v}\right)>\frac{\rho_{\nu}}{2-\rho_{v}} N_{v}(2 R) \quad\left(R \geq R_{0}^{(v)}\right)
$$

where

$$
N_{v}(r)=N\left(r, 0, f_{v}\right)+N\left(r, \infty, f_{v}\right) \quad(v=1, \ldots, n) .
$$

Thus, by putting $\rho=\min _{\nu} \rho_{\nu}$, we have

$$
N\left(2 R, 0, f_{v}\right)-N\left(2 R, \infty, f_{v}\right)>\frac{\rho}{2-\rho} N_{\nu}(2 R) \quad\left(R \geq R_{0}^{(v)}\right) .
$$


Applying Lemma 1 for $\varphi(z)=f_{v}(z)$ and in view of (16), we have

$$
\left|\frac{\log \left|f_{v}(z)\right|}{N\left(2 R, 0, f_{v}\right)-N\left(2 R, \infty, f_{v}\right)}-1\right| \leq\left(1+\log \frac{4 e}{\eta_{\nu}}\right) \frac{2-\rho}{\rho} \frac{Q_{0}^{(v)}(2 R)}{N_{v}(2 R)}
$$

in $|z|<R$, outside a set of circles the sum of whose radii is at most $2 \eta_{\nu} R$ provided $R \geq R_{0}^{(v)}$ and $0<\eta_{\nu}<\frac{8}{3} e$.

Let $\varepsilon(>0)$ be given. By Lemma 2 it is possible to choose an arbitrarily large number $R_{\varepsilon}^{(v)}$ such that

$$
\left(1+\log \frac{4 e}{\eta_{v}}\right) \frac{2-\rho}{\rho} \frac{Q_{0}^{(v)}(r)}{N_{v}(r)}<\varepsilon
$$

for each $v(1 \leq v \leq n)$ in a set $E\left(\eta_{v}, \varepsilon\right)$ of $r\left(<R_{\varepsilon}^{(v)}\right)$ of measure at least $\left(1-2 \eta_{v}\right) R_{\varepsilon}^{(v)}$.

Then we have, by setting $R_{\varepsilon}=\min R_{\varepsilon}^{(v)}$, in view of (29)-(32), and using the first fundamental theorem,

$$
\left|\frac{\log \left|f_{v}(z)\right|}{m\left(r, f_{v}\right)}-1\right|<\varepsilon \quad\left(|z|<r<R_{\varepsilon}\right)
$$

in each set $E\left(\eta_{\nu}, \varepsilon\right)$ of $r$.

Thus we deduce

$$
(1-\varepsilon) m\left(r, f_{v}\right)<\log \left|f_{v}(z)\right|<(1+\varepsilon) m\left(r, f_{v}\right) \quad(v=1,2, \ldots, n)
$$

by (33) in a set

$$
E(\eta, \epsilon)=\bigcap_{\nu} E\left(\eta_{\nu}, \varepsilon\right)
$$

We have then from the right-hand side of (34), by taking the maximum for $v$,

$$
\max _{v} \log \left|f_{v}(z)\right|<(1+\varepsilon) \max _{v} m\left(r, f_{v}\right)
$$

and further, by taking the supremum at $|z|=r(r \in E(\eta, \varepsilon))$ of both sides of the above inequality, we have by using Lemma 8 ,

$$
\log M(r, f)-O(1)=\sup _{|z|=r} \max _{v} \log \left|f_{v}(z)\right| \leq(1+\varepsilon) \max _{v} m\left(r, f_{v}\right) .
$$

Similarly we have from the left-hand side of (34), in view of Lemma 8,

$$
\log L(r, f)+O(1)=\inf _{|z|=r} \max _{v} \log \left|f_{v}(z)\right| \geq(1-\varepsilon) \max _{v} m\left(r, f_{v}\right) .
$$


Hence we deduce by using the above two inequalities

$$
\frac{1-\varepsilon}{1+\varepsilon} \leq \frac{(1+o(1)) \log L(r, f)}{(1-o(1)) \log M(r, f)} \leq 1
$$

in a set $E(\eta, \varepsilon)$.

Therefore, it follows from (35) that

$$
\begin{aligned}
\frac{1-\varepsilon}{1+\varepsilon} \frac{\max _{v} m\left(r, f_{v}\right)}{T(r, f)} & \leq \frac{(1+o(1)) \log L(r, f)}{T(r, f)} \\
& \leq \frac{(1-o(1)) \log M(r, f)}{T(r, f)} \leq \frac{1+\varepsilon}{1-\varepsilon} \frac{\max _{v} m\left(r, f_{v}\right)}{T(r, f)}
\end{aligned}
$$

in $E(\eta, \varepsilon)$.

Since $\eta$ and $\varepsilon$ are arbitrary, we conclude that

$$
\log L(r, f) \sim \log M(r, f)
$$

from (35) too, which proves (5).

Also we deduce from the above inequality (36) that

$$
\begin{aligned}
\liminf _{r \rightarrow \infty} \frac{\max _{v} m\left(r, f_{v}\right)}{T\left(r, f_{v}\right)} & \leq \liminf _{r \rightarrow \infty} \frac{\log L(r, f)}{T(r, f)} \\
& \leq \limsup _{r \rightarrow \infty} \frac{\log M(r, f)}{T(r, f)} \leq \limsup _{r \rightarrow \infty} \frac{\max _{v} m\left(r, f_{v}\right)}{T(r, f)}
\end{aligned}
$$

in a set of $r$ of upper density 1.

In view of (18), we now have

$$
\begin{aligned}
\liminf _{r \rightarrow \infty} \frac{\max _{v} m\left(r, f_{v}\right)}{T(r, f)} & \geq \liminf _{r \rightarrow \infty} \frac{\frac{1}{n} \sum_{v=1}^{n} m\left(r, f_{v}\right)}{T(r, f)} \geq \liminf _{r \rightarrow \infty} \frac{m(r, f)-O(1)}{T(r, f)} \\
& =\delta(\infty, f) .
\end{aligned}
$$

Similarly we get, in view of (19),

$$
\limsup _{r \rightarrow \infty} \frac{\max _{v} m\left(r, f_{v}\right)}{T(r, f)} \leq \limsup _{r \rightarrow \infty} \frac{n m(r, f)+O(1)}{T(r, f)}=n \Delta(\infty, f) .
$$

Thus we have, by combining (39) and (40) with (38),

$$
\delta(\infty, f) \leq \liminf _{r \rightarrow \infty} \frac{\log L(r, f)}{T(r, f)} \leq \limsup _{r \rightarrow \infty} \frac{\log M(r, f)}{T(r, f)} \leq n \Delta(\infty, f) .
$$

This proves the inequality (6). Consequently, (7) is yielded obviously. 
Next if $f_{v}(z)$ does not satisfy the asymptotic relation (29), we choose $\gamma_{v}(\neq 0)$ such that

$$
N\left(r, \frac{1}{f_{v}-\gamma_{v}}\right) \sim T\left(r, f_{v}\right) \quad \text { and } \quad f_{v}(0) \neq \gamma_{\nu} \quad(v=1,2, \ldots, n) .
$$

Put $F_{v}(z)=c_{\nu}\left\{f_{v}(z)-\gamma_{\nu}\right\}$, where $F_{\nu}(0)=1$. Then we have

$$
T\left(r, f_{\nu}\right) \sim T\left(r, F_{\nu}\right) \sim N\left(r, \frac{1}{F_{0}}\right) \text { and } N\left(r, f_{\nu}\right)=N\left(r, F_{\nu}\right),
$$

consequently $m\left(r, f_{v}\right) \sim m\left(r, F_{v}\right)$.

Thus the hypotheses in the theorem and the additional property hold with $f_{v}(z)$ replaced by $F_{v}(z)$. Hence the conclusion of the theorem holds with $f(z)$. This proves the general validity of the theorem.

\section{Proof of Theorem 2}

A discussion similar to that for Theorem 1 yields

$$
\log L(r, f) \sim \log M(r, f)
$$

in view of (35) since $\varepsilon$ is arbitrary, in a set of $r$ of upper density 1.

Further, (36) in Theorem 1 shows that

$$
\begin{aligned}
\frac{1-\varepsilon}{1+\varepsilon} \frac{\max _{v} m\left(r, f_{v}\right)}{T(r, f)} & \leq \frac{(1+o(1)) \log L(r, f)}{T(r, f)} \\
& \leq \frac{(1-o(1)) \log M(r, f)}{T(r, f)} \leq \frac{1+\varepsilon}{1-\varepsilon} \frac{\max _{v} m\left(r, f_{v}\right)}{T(r, f)}
\end{aligned}
$$

in $E(\eta, \varepsilon)$.

The left-hand side of (41) is

$$
\begin{aligned}
\liminf _{r \rightarrow \infty} \frac{\max _{v} m\left(r, f_{v}\right)}{T(r, f)} & \geq \liminf _{r \rightarrow \infty} \frac{\frac{1}{n} n \max _{v} m\left(r, f_{v}\right)}{T(r, f)} \\
& \geq \liminf _{r \rightarrow \infty} \frac{\frac{1}{n} \sum_{v=1}^{n} m\left(r, f_{v}\right)}{T(r, f)} \\
& \geq \liminf _{r \rightarrow \infty} \frac{m(r, f)-O(1)}{T(r, f)} \\
& =\delta(\infty, f) \\
& \geq \max _{v} \delta\left(\infty, f_{v}\right)
\end{aligned}
$$

by using (22) in Lemma 5. 
The right-hand side of (41) is

$$
\begin{aligned}
\liminf _{r \rightarrow \infty} \frac{\max _{v} m\left(r, f_{v}\right)}{T(r, f)} & \leq \liminf _{r \rightarrow \infty} \frac{n m(r, f)+O(1)}{T(r, f)} \\
& =n \liminf _{r \rightarrow \infty} \frac{m(r, f)}{T(r, f)} \\
& =n \delta(\infty, f) \leq n^{2} \max _{v} \delta\left(\infty, f_{v}\right)
\end{aligned}
$$

in view of (19) and (24).

Thus we obtain the desired result, which proves Theorem 2 .

\section{Proof of Theorem 3}

Similarly by using (20), the left-hand side is

$$
\begin{aligned}
\limsup _{r \rightarrow \infty} \frac{\max _{v} m\left(r, f_{v}\right)}{T(r, f)} & \geq \limsup _{r \rightarrow \infty} \frac{\frac{1}{n} n \max _{v} m\left(r, f_{v}\right)}{T(r, f)} \\
& \geq \limsup _{r \rightarrow \infty} \frac{\frac{1}{n} \sum_{v=1}^{n} m\left(r, f_{v}\right)}{T(r, f)} \\
& \geq \limsup _{r \rightarrow \infty} \frac{m(r, f)-O(1)}{T(r, f)}=\Delta(\infty, f) \\
& \geq \Delta\left(\infty, f_{v}\right) \quad(v=1,2, \ldots, n)
\end{aligned}
$$

in view of (23).

Thus

$$
\limsup _{r \rightarrow \infty} \frac{\max _{v} m\left(r, f_{v}\right)}{T(r, f)} \geq \max _{\nu} \limsup _{r \rightarrow \infty} \frac{m\left(r, f_{v}\right)}{T\left(r, f_{v}\right)}=\max _{v} \Delta\left(\infty, f_{v}\right) .
$$

In view of (21), the right-hand side is

$$
\begin{aligned}
\limsup _{r \rightarrow \infty} \frac{\max _{v} m\left(r, f_{v}\right)}{T(r, f)} & \leq \limsup _{r \rightarrow \infty} \frac{\max _{v} m\left(r, f_{v}\right)}{\frac{1}{n} T\left(r, f_{v}\right)-O(1)} \quad(v=1,2, \ldots, n) \\
& =n \limsup _{r \rightarrow \infty} \frac{\max _{v} m\left(r, f_{v}\right)}{T\left(r, f_{v}\right)} \\
& =n \max _{v} \limsup _{r \rightarrow \infty} \frac{m\left(r, f_{v}\right)}{T\left(r, f_{v}\right)} \\
& =n \max _{v} \Delta\left(\infty, f_{v}\right) .
\end{aligned}
$$


Thus we deduce from (41)

$$
\max _{\nu} \Delta\left(\infty, f_{v}\right) \leq \limsup _{r \rightarrow \infty} \frac{\log L(r, f)}{T(r, f)} \leq \limsup _{r \rightarrow \infty} \frac{\log M(r, f)}{T(r, f)} \leq n \max _{\nu} \Delta\left(\infty, f_{v}\right) .
$$

This proves Theorem 3 .

\section{Proofs of Corollaries 1 and 2}

Proof of Corollary 1. It follows from (22) that

$$
\sum_{\nu=1}^{n} \delta\left(\infty, f_{\nu}\right) \leq \sum_{\nu=1}^{n} \delta(\infty, f)=n \delta(\infty, f)
$$

then we have by combining the right-hand side of (8) and the left-hand side of (6)

$$
\delta(\infty, f) \leq \liminf _{r \rightarrow \infty} \frac{\log L(r, f)}{T(r, f)} \leq \liminf _{r \rightarrow \infty} \frac{\log M(r, f)}{T(r, f)} \leq n \delta(\infty, f) .
$$

This proves (10).

Proof of Corollary 2. It follows from (26) that

$$
\Delta(\infty, f) \leq n \max _{v} \Delta\left(\infty, f_{v}\right)
$$

Then we have by combining the left-hand side of (9) and the right-hand side of (6)

$$
\frac{1}{n} \Delta(\infty, f) \leq \limsup _{r \rightarrow \infty} \frac{\log L(r, f)}{T(r, f)} \leq \limsup _{r \rightarrow \infty} \frac{\log M(r, f)}{T(r, f)} \leq n \Delta(\infty, f) .
$$

This proves (11).

\section{Example}

If the hypothesis $\delta(\infty, f)>0$ is omitted, the conclusion of Theorem 1 is no longer true. For this reason $\delta(\infty, f)>0$ is the essential condition for Theorem 1 .

For instance, consider the functions

$$
A_{0}(z)=\prod\left(1-\frac{z}{e^{n}}\right), \quad A_{1}(z)=\prod\left(1+\frac{z}{e^{n}}\right)
$$

and put

$$
\varphi(z)=\frac{A_{1}(z)}{A_{0}(z)}
$$


Then $\varphi(z)$ is of order zero and obviously

$$
\log L(r, \varphi)=-\log M(r, \varphi)
$$

Let $f(z)$ be an algebroid function defined by

$$
F(z, f) \equiv A_{0}(z) f^{n}-A_{1}(z) f^{n-1}+A_{1}(z)-A_{0} e^{i \theta}=0 \quad(\theta \text { is irrational })
$$

which is irreducible.

The irreducibility of this equation (45) can be showed by the following.

Suppose that

$$
A_{0} f^{n}-A_{1} f^{n-1}+A_{1}-A_{0} e^{i \theta} \equiv A(z, f) B(z, f) .
$$

When $n=2$, the irreducibility of (46) is obvious. When $n=3$, suppose that

$$
A_{0} f^{3}-A_{1} f^{2}+A_{1}-A_{0} e^{i \theta}=\left(\alpha_{2} f^{2}+\alpha_{1} f+\alpha_{0}\right)\left(\beta_{1} f+\beta_{0}\right),
$$

then we have

$$
\alpha_{0} \beta_{0}=A_{1}-A_{0} e^{i \theta}, \quad \alpha_{1} \beta_{0}+\alpha_{0} \beta_{1}=0, \quad \alpha_{2} \beta_{0}+\alpha_{1} \beta_{1}=-A_{1} .
$$

Hence there is some suitable function $k$ which satisfies

$$
\alpha_{1}=k \alpha_{0}, \quad \beta_{1}=-k \beta_{0}
$$

from the second equation, where $\alpha_{0}, \beta_{0}$ are prime for each other. Then we deduce the identity

$$
\alpha_{2} \beta_{0}-k^{2} \alpha_{0} \beta_{0}=-A_{1}
$$

From the third equation which shows that $A_{1}$ is a multiple of $\beta_{0}$. But the zeros of $\beta_{0}$ are the zeros of $A_{1}-A_{0} e^{i \theta}$ from the first equation, which is a contradiction.

Thus equation (46) is irreducible.

For general $n \in \mathbb{N}$, take $m, l$ such that $m \geq l$ and $m+l=n$, and

$$
\begin{aligned}
A_{0} f^{n}-A_{1} f^{n-1}+A_{1}-A_{0} e^{i \theta}= & \left(\alpha_{m} f^{m}+\alpha_{m-1} f^{m-1}+\cdots+\alpha_{1} f+\alpha_{0}\right) \\
& \times\left(\beta_{l} f^{l}+\beta_{l-1} f^{l-1}+\cdots+\beta_{1} f+\beta_{0}\right) .
\end{aligned}
$$


Then we have $n+1$ identities as follows:

$$
\begin{aligned}
& \alpha_{0} \beta_{0}=A_{1}-A_{0} e^{i \theta}, \\
& \alpha_{1} \beta_{0}+\alpha_{0} \beta_{1}=0, \\
& \alpha_{2} \beta_{0}+\alpha_{1} \beta_{1}+\alpha_{0} \beta_{2}=0, \\
& \alpha_{3} \beta_{0}+\alpha_{2} \beta_{1}+\alpha_{1} \beta_{2}+\alpha_{0} \beta_{3}=0, \\
& \ldots \\
& \alpha_{m} \beta_{0}+\alpha_{m-1} \beta_{1}+\alpha_{m-2} \beta_{2}+\cdots+\alpha_{m-l} \beta_{l}=0, \\
& \alpha_{m} \beta_{1}+\alpha_{m-2} \beta_{2}+\cdots+\alpha_{m-l+1} \beta_{l}=0, \\
& \ldots \\
& \alpha_{m} \beta_{l-2}+\alpha_{m-1} \beta_{l-2}+\alpha_{m-2} \beta_{l}=0, \\
& \alpha_{m} \beta_{l-1}+\alpha_{m-1} \beta_{l}=-A_{1}, \\
& \alpha_{m} \beta_{l}=A_{0} .
\end{aligned}
$$

Hence similarly we get

$$
\alpha_{1}=k \alpha_{0}, \quad \beta_{1}=-k \beta_{0}
$$

from (48), where $\alpha_{0}, \beta_{0}$ are prime for each other, and $k$ is some suitable function. Then we deduce the identity

$$
\alpha_{2} \beta_{0}-k^{2} \alpha_{0} \beta_{0}+\alpha_{0} \beta_{2}=0
$$

from (49), and we get

$$
\alpha_{2}=K_{2} \alpha_{0}, \quad \beta_{2}=K_{2}^{\prime} \beta_{0}
$$

where $K_{2}, K_{2}^{\prime}$ are some suitable functions.

By using (54) and (55) with (50), we can obtain

$$
\alpha_{3}=K_{3} \alpha_{0}, \quad \beta_{3}=K_{3}^{\prime} \beta_{0}
$$

where $K_{3}, K_{3}^{\prime}$ are some suitable functions.

Now we repeat the same procedure to the identity (51), we can deduce at last

$$
\alpha_{j}=K_{j} \alpha_{0} \quad(j=1,2, \ldots, m), \quad \beta_{j}=K_{j}^{\prime} \beta_{0} \quad(j=1,2, \ldots, l)
$$

where $K_{j}, K_{j}^{\prime}$ are suitable functions.

Thus we have the identity

$$
K_{m} \alpha_{0} K_{l-1}^{\prime} \beta_{0}+K_{m-1} \alpha_{0} K_{l}^{\prime} \beta_{0}=-A_{1},
$$

from (52) which shows that $A_{1}$ is a multiple of $\alpha_{0} \beta_{0}$. 
Hence it follows also that $A_{0}$ is a multiple of $\alpha_{0} \beta_{0}$ from (47) or (53), which is a contradiction.

Next we consider the case that $\beta_{0}$ may be zero-free, for example a constant. When $n=3$, suppose that

$$
A_{0} f^{3}-A_{1} f^{2}+A_{1}-A_{0} e^{i \theta}=\left(\alpha_{2} f^{2}+\alpha_{1} f+\alpha_{0}\right)\left(\beta_{1} f+\beta_{0}\right),
$$

then we have

$$
\alpha_{2} \beta_{1}=A_{0}, \quad \alpha_{2} \beta_{0}+\alpha_{1} \beta_{1}=-A_{1}, \quad \alpha_{1} \beta_{0}+\alpha_{0} \beta_{1}=0, \quad \alpha_{0} \beta_{0}=A_{1}-A_{0} e^{i \theta} .
$$

Suppose that $\beta_{0}=c$, so we have

$$
\alpha_{1}=-\frac{\beta_{1}}{c} \alpha_{0}
$$

from the third equation.

From the first equation we have

$$
c \alpha_{0}=A_{1}-A_{0} e^{i \theta}=A_{1}-\alpha_{2} \beta_{1} e^{i \theta}
$$

by using the fourth equation.

We have from the second equation that $\alpha_{2}=-\left(A_{1}+\alpha_{1} \beta_{1}\right) / c$, so then we have

$$
c \alpha_{0}=A_{1}+\frac{A_{1}+\alpha_{1} \beta_{1}}{c} \beta_{1} e^{i \theta} .
$$

Hence we get

$$
c \alpha_{0}+\frac{\alpha_{0} \beta_{0}^{3}}{c} e^{i \theta}=\left(1+\frac{\beta_{1}}{c} e^{i \theta}\right) A_{1}
$$

in view of (57), and consequently

$$
\frac{\alpha_{0}}{c}\left(c^{3}+\beta_{1}^{3} e^{i \theta}\right)=\left(c+\beta_{1} e^{i \theta}\right) A_{1},
$$

where $c^{3}+\beta_{1}^{3} e^{i \theta}, c+\beta_{1} e^{i \theta}$ are prime for each other. This shows that $A_{1}$ is a multiple of $\alpha_{0}$. Thus we have a contradiction from the fourth equation.

For the general case, we use $n+1$ identities (47)-(53) similarly. Firstly we have

$$
\alpha_{1}=-\frac{\beta_{1}}{c} \alpha_{0}
$$

from (48), and we then get

$$
\alpha_{1}=K_{1} \alpha_{0}
$$


where $K_{1}$ is some suitable function. Then we have from (49)

$$
\alpha_{2}=\left(\frac{\beta_{1}^{2}}{c^{2}}-\beta_{2}\right) \alpha_{0} \equiv K_{2} \alpha_{0}
$$

Similarly we have from (50)

$$
\alpha_{3}=\left(\frac{\beta_{1}}{c^{2}}-\frac{\beta_{1}^{2}}{c^{3}}+\frac{\beta_{2}}{c}-\frac{\beta_{3}}{c}\right) \alpha_{0} \equiv K_{3} \alpha_{0}
$$

where $K_{2}, K_{3}$ are some suitable functions.

By using (51), similarly we can get

$$
\alpha_{j}=K_{j} \alpha_{0} \quad(j=1,2, \ldots, n),
$$

where $K_{j}$ is some suitable function.

Hence we have from (52) by using (58)

$$
K_{m} \alpha_{0} \beta_{l-1}+K_{m-1} \alpha_{0} \beta_{l}=-A_{1},
$$

i.e.

$$
\alpha_{0}\left(K_{m} \beta_{l-1}+K_{m-1} \beta_{l}\right)=-A_{1},
$$

which shows that $A_{1}$ is a multiple of $\alpha_{0}$.

On the other hand, from (53) we have

$$
K_{m} \alpha_{0} \beta_{l}=A_{0}
$$

which shows $A_{0}$ is a multiple of $\alpha_{0}$ also, which is a contradiction.

Consequently, this contradiction of (46) gives us that equation (45) is irreducible.

Now we consider

$$
F(z, f)=A_{0} f^{n}-A_{1} f^{n-1}+A_{1}-A_{0} e^{i \theta}=0
$$

and

$$
a_{j}=\exp \left[\frac{2 \pi j}{n} i+\frac{\theta}{n} i\right] \quad(j=1,2, \ldots, n)
$$

Then we have

$$
F\left(z, a_{j}\right)=\left(1-\exp \left(\frac{n-1}{n} 2 \pi j i-\frac{n-1}{n} \theta i\right)\right) A_{1} \equiv \lambda_{j} A_{1} \quad\left(\lambda_{j} \neq 0\right) .
$$


Hence $F\left(z, a_{j}\right)=0$ yields $A_{1}=0(j=1,2, \ldots, n)$. Then since

$$
g_{j}(z)=F\left(z, a_{j}\right)=\lambda_{j} A_{1},
$$

we have, in view of (44),

$$
f_{j}(z)=\frac{g_{j}(z)}{A_{0}(z)}=\frac{\lambda_{j} A_{1}(z)}{A_{0}(z)}=\lambda_{j} \varphi \quad(j=1,2, \ldots, n) .
$$

Then we also have

$$
\begin{aligned}
& N\left(r, a_{\nu}, f\right)=\frac{1}{n} N\left(r, 0, g_{\nu}\right)=\frac{1}{n} N\left(r, 0, A_{1}\right), \\
& N(r, \infty, f)=\frac{1}{n} N\left(r, 0, A_{0}\right)=\frac{1}{n} N(r, \varphi) .
\end{aligned}
$$

We note that

$$
\begin{aligned}
T(r, f)+O(1)=\mu(r, A) & =\frac{1}{2 \pi n} \int_{0}^{2 \pi} \log \max \left\{1,\left|A_{0}\right|,\left|A_{1}\right|\right\} d \theta \\
& \sim \frac{1}{n} T\left(r, \frac{A_{1}}{A_{0}}\right)=\frac{1}{n} T(r, \varphi) .
\end{aligned}
$$

This shows at once that $f(z)$ is of order zero.

Now we estimate $m(r, \varphi)$ of function

$$
\varphi(z)=\frac{A_{1}(z)}{A_{0}(z)}=\prod_{n=1}^{\infty} \frac{1+\left(z / e^{n}\right)}{1-\left(z / e^{n}\right)} .
$$

Since $\varphi(z)=A_{1}(z) / A_{0}(z)$ satisfies a $q$-difference equation

$$
(1-q z) \varphi(q z)=(1+q z) \varphi(z) \quad\left(q=\frac{1}{e}\right)
$$

hence [1]

$$
m(r, f)=o(\log r)^{2}
$$

On the other hand, since

$$
n(r, \varphi) \sim \log r
$$

we have

$$
N(r, \varphi)=\int_{1}^{r} \frac{\log t}{t} d t=\frac{1}{2}(\log r)^{2}(1+o(1)) .
$$

Hence we deduce

$$
T(r, \varphi)=\frac{1}{2}(\log r)^{2}(1+o(1))
$$


Thus this yields

$$
\delta(\infty, f)=\Delta(\infty, f)=0
$$

Also, in view of (59),

$$
\begin{aligned}
\log M(r, f) & =\sup _{|z|=r} \max _{v}\left\{\log \left|f_{1}\right|, \log \left|f_{2}\right|, \ldots, \log \left|f_{n}\right|\right\} \\
& =\sup _{|z|=r}(\log |\varphi|+O(1)) \\
& =\sup _{|z|=r} \log |\varphi|+O(1) \\
& =\log M(r, \varphi)=-\log L(r, \varphi)=-\inf _{|z|=r} \log |\varphi|+O(1) \\
& =-\log L(r, f)
\end{aligned}
$$

which means consequently that (5) does not hold.

Acknowledgement. The author wishes to express his thanks to the referee for many useful comments and suggestions.

\section{REFERENCES}

[1] W. Bergweiler, K. Ishizaki and N. Yanagihara. Growth of meromorphic solutions of some functional equations. Aeq. Math. To appear.

[2] R. P. Boas. Entire Functions. Academic Press, New York, 1954.

[3] M. L. Cartwright. Intergral Functions. Cambridge University Press, 1956.

[4] A. Edrei and W. H. J. Fuchs. The deficiencies of meromorphic functions of order less than one. Duke Math. J. 27 (1960), 233-249.

[5] W. K. Hayman. Meromorphic Functions. Oxford University Press, Oxford, 1964.

[6] P. Griffiths and J. King. Nevanlinna theory and holomorphic mappings between algebraic varieties. Acta Math. 130 (1973), 145-220.

[7] Y. Kubota. On mermorphic functions of order zero. Kodai Math. Sem. Rep. 21 (1969), 405-412.

[8] R. Nevanlinna. Eindeutige analytische Funktionen. Springer, Berlin, 1953.

[9] I. V. Ostrovskii. On defects of meromorphic functions with lower order less than one. Sov. Math.Dokl. 4 (1963), 587-591.

[10] M. Ozawa. Deficiencies of an algebroid function. Kodai Math. Sem. Rep. 21 (1969), 262-276.

[11] A. Rauch. Remarques sur les fonctions algébroïdes entières. Bull. Sci. Math. 59 (1935), 8-26.

[12] A. Sadullaev. Deficient divisors in the Valiron sense. Math. USSR Sbornik 36(4) (1980), 535-547.

[13] T. Sato. On the asymptotic behaviour of algebroid functions of extremal growth. Kodai Math. J. 5 (1982), 145-153.

[14] H. Selberg. Über die Wertverteilung der algebroiden Funktionen. Math. Zeit. 31 (1930), 1-71. 
[15] Y. Tsumura. On algebroïd functions. J. Japan Math. Phys. Society 15 (1941), 77-96.

[16] G. Valiron. Sur la dérivée des fonctions algébroides. Bull. Soc. Math. France 59 (1931), 17-39.

[17] Valiron, G. Sur les valeurs deficientes des fonctions algébroides méromorphes d'ordre nul. J. d'Analyse Math. 1 (1951), 28-42.

[18] H.-H. Wu. The Equidistribution Theory of Holomorphic Curves (Ann. Math. Studies 64). Princeton University Press, Princeton, NJ, 1970.

Tsuneo Sato

Faculty of Mathematics

Chiba University

1-33 Yayoi-cho, Image-ku

Chiba 263-8522

Japan 